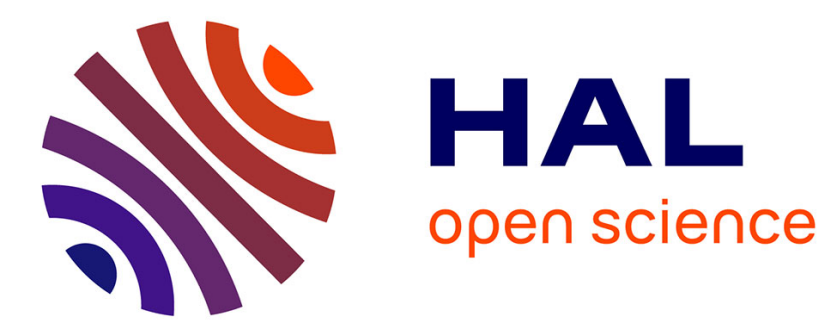

\title{
Experiments and modeling of an unsteady turbulent channel flow
}

Sedat F. Tardu, Paulo da Costa

\section{To cite this version:}

Sedat F. Tardu, Paulo da Costa. Experiments and modeling of an unsteady turbulent channel flow. AIAA Journal, 2005, 43 (1), pp.140-149. 10.2514/1.6332 . hal-00262006

\section{HAL Id: hal-00262006 https://hal.science/hal-00262006}

Submitted on 7 Feb 2020

HAL is a multi-disciplinary open access archive for the deposit and dissemination of scientific research documents, whether they are published or not. The documents may come from teaching and research institutions in France or abroad, or from public or private research centers.
L'archive ouverte pluridisciplinaire HAL, est destinée au dépôt et à la diffusion de documents scientifiques de niveau recherche, publiés ou non, émanant des établissements d'enseignement et de recherche français ou étrangers, des laboratoires publics ou privés. 


\title{
Experiments and Modeling of an Unsteady Turbulent Channel Flow
}

\author{
Sedat F. Tardu* and Paulo da Costa ${ }^{\dagger}$ \\ Laboratoire des Ecoulements Géophysiques et Industriels, 38041 Grenoble Cédex, France
}

\begin{abstract}
The $k-\omega$ model is combined with the rapid distortion scheme to develop effective unsteady closures in nonequilibrium wall flows subject to oscillating shear. The phase-averaged eddy viscosity is related to the modulation of the effective strain parameter whose distribution is obtained from the steady turbulent channel flow data. New experimental data on the wall and Reynolds shear stresses are reported. The model predicts the time-mean and phase-averaged flow quantities in a satisfactory way when the imposed frequency is smaller than the median frequency of the near-wall turbulence in the relaxation regime. It is particularly successful at the wall and in the low buffer layer.
\end{abstract}

\section{Introduction}

$\mathbf{T}$ HE aim of this study is to develop effective unsteady closures for the wall flows subject to imposed unsteadiness. Such flows are in temporal nonequilibrium. They occur in practice on helicopter blades, in turbomachinery, in internal combustion systems, etc. The forcing can simply be achieved by a time-varying flow rate or pressure gradient. Considerable experimental efforts have been made in the past to explore the different physical aspects of unsteady turbulent flows. Several groups involved in this subject, and Tu and Ramaprian, ${ }^{1}$ Mao and Hanratty, ${ }^{2}$ Brereton et al., ${ }^{3}$ and Tardu et al. ${ }^{4}$ are only a few of them.

The problem we are interested in is schematically shown in Fig. 1. A fully developed turbulent channel flow is forced by imposed velocity oscillations at the centerline. The velocity oscillations induce oscillating shear near the wall. The flow is in time equilibrium during the oscillation period when the imposed frequency is low enough. This is called the quasi-steady regime in this paper. Any closure scheme that is successful in steady turbulent internal flows would predict well the flow behavior in this regime. The oscillating vorticity diffuses to a distance $\Delta y \approx \sqrt{ }(\nu T)$ from the wall during the oscillation period $T$ with $v$ standing for the viscosity. Viscous diffusion alone governs the removal of unsteady vorticity from the wall, when $\Delta y$ is mainly constrained within the low buffer layer of thickness $12\left(v / \bar{u}_{\tau}\right)$, where $\bar{u}_{\tau}$ is the shear velocity. That gives the condition $\sqrt{ } T^{+}=\sqrt{ }\left[T /\left(v / \bar{u}_{\tau}^{2}\right)\right]<12$ in wall units. Thus, the turbulence does not participate in the diffusion when $\Delta y^{+} \approx \sqrt{ } T^{+}<12$ because at distances from the wall where turbulent diffusion becomes important there is no vorticity left to diffuse. Slightly modifying the length scale by introducing the thickness of the viscous Stokes layer $l_{s}=\sqrt{ }(v T / \pi)$ results in $l_{s}^{+}=l_{s} /\left(v / \bar{u}_{\tau}\right)<7$. That is, the condition for the shear wave generated at the wall to reach the asymptotic outer values before the turbulence can play an appreciable role in the momentum transfer. Experiments show that the long time-averaged mean flow is not affected by the imposed unsteadiness, even when the imposed amplitude and frequency are so large that reverse flow appears near the wall. This is because the streaming terms, such as the mean of oscillating shear stress $\widetilde{u^{\prime} v^{\prime}}$ occurring in the time-mean equations are negligible. Thus, if the imposed frequency is high enough one has the coexistence of an unaltered time mean and a viscous oscillating Stokes-type flow. The

\footnotetext{
*Associate Professor, B.P. 53X; Sedat.Tardu@ @mg.inpg.fr.
}

${ }^{\dagger}$ Research Assistant, B.P. 53X. frequency delimitating this regime is close to the median frequency of the near-wall turbulence. Consequently, the modulation of the turbulent quantities weakens, and large time lags with respect to the centerline velocity appear because of the finite turbulence response time. This is called the relaxation regime here.

Although this physical process is rather easy to understand, the modeling of unsteady wall flows is not obvious. Most of the researchers have directly applied the phase-averaged version of existing closures, by taking more or less into account the real nonequilibrium character of the problem. We can quote Fan et al. ${ }^{5}$ and Chernobrovkin and Lakshminarayana, ${ }^{6}$ who applied the $k-\varepsilon$ scheme; Ekaterinaris et al., ${ }^{7}$ who used three different versions of the $k-\omega$ method combined with the baseline and shear-stress transport models; and Srinivasan et al., ${ }^{8}$ who chose algebraic models and renormalization group analysis. The majority of the models found in the literature use quasi-steady assumptions. An instantaneous equilibrium is supposed between, for example, the phase average of the eddy viscosity and $\langle k\rangle^{2} /\langle\varepsilon\rangle$ or $\langle k\rangle /\langle\omega\rangle$, despite the fact that large phase shifts can appear between these quantities, in particular in the high imposed frequency regime. Truly unsteady effects have been tentatively taken into account in Mankbadi and $\mathrm{Liu}^{9}$ and to some extend in Mao and Hanratty ${ }^{2}$ and in Greenblatt. ${ }^{10}$

The first aim of this investigation is to provide further experimental data in turbulent channel flows subject to imposed velocity oscillations. Measurements on the wall and Reynolds shear stresses are conducted in a wide range of imposed frequency. They are discussed and compared with existing data. The second aim is to develop an unsteady one-point closure based on the classical $k-\omega$ scheme ${ }^{11}$ combined with a rapid distortion theory approach. This approach originally given by Townsend ${ }^{12}$ and analyzed and developed by Maxey ${ }^{13}$ has been efficiently applied to unsteady wall flows by Mankbadi and $\mathrm{Liu}^{9}$ (MK hereafter) through simplified closure assumptions and gave good qualitative (although not quantitative) agreement compared with experimental data. The target here is to go a step further and see if with a strategically similar though technically different methodology we can also obtain satisfactory quantitative agreement. Emphasis is put on the wall shear-stress turbulent intensity and Reynolds shear-stress modulation characteristics that are quite sensitive to the modeling procedure.

\section{Experimental Setup and Data Reduction}

The experiments were performed in a fully developed turbulent water channel $(100 \mathrm{~mm}$ wide and $2600 \mathrm{~mm}$ long, the aspect ratio is $10 / 1$ ). The time mean centerline velocity was $U_{c}=17.5 \mathrm{~cm} / \mathrm{s}$ corresponding to the Reynolds number $8.5 \times 10^{3}$ based on the halfchannel height. A specifically designed pulsating device produces the oscillations in the flow rate. The mean flow, the period, and the amplitude of the imposed velocity oscillations can be independently changed. The reader is referred to Tardu et al. ${ }^{4}$ for further details 


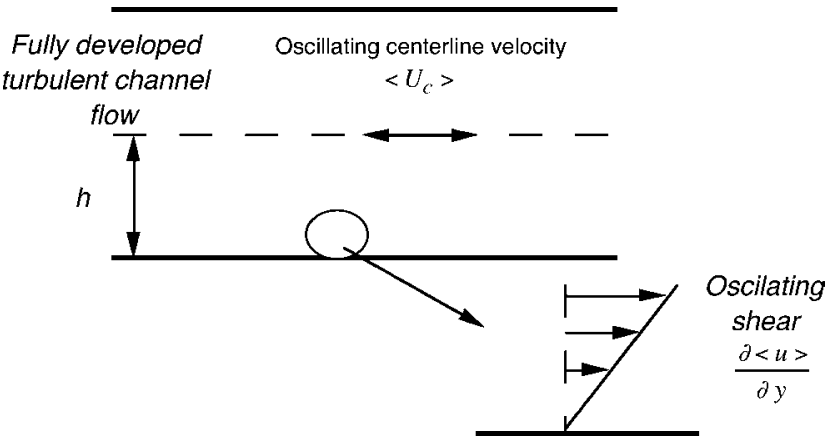

Fig. 1 Schematic picture of the problem.

concerning the flow conditions and related discussions. Simultaneous measurements of the instantaneous streamwise $u(t)$ and wall normal $v(t)$ velocities were performed by means of a TSI $2148-10 \mathrm{w}$ $\mathrm{x}$-hot-film probe of seven wall units in the wall normal direction. The probe was calibrated vs velocity and angle. The look-up table technique ${ }^{14}$ allowed the determination of $u(t)$ and $v(t)$. A DISA 55 R11 single hot film with a sensing length $\Delta z=9\left(v / \bar{u}_{\tau}\right)$ was used for both comparison and streamwise velocity measurements near and in the viscous sublayer. The wall shear-stress measurements are performed by means of a flush-mounted TSI-1268 W hot film at the wall of $8\left(v / \bar{u}_{\tau}\right)$ spanwise extend. The vertical probe displacements were made using a controlled traversing mechanism. The hot films were operated in the constant temperature mode with 5 to $8 \%$ overheat by AHARONI AN-1003 anemometer units. The calibrations were performed done in situ as described by Tardu et al., ${ }^{4}$ wherein the frequency response of hot-film gauges and other experimental details are discussed in detail. The shear-stress measurements reported here are new, and they compare reasonably well with our previous investigations. ${ }^{15}$

Data acquisition was performed on a PC computer with a 16-bit accuracy. The sampling frequency was $f_{S}=3 u_{\tau}(2 / v)$. The signal was low-pass filtered by a Krohn Hite filter at $f_{S} / 2$. The total duration of each record was $T_{r}=10^{5}\left(h / U_{c}\right)$, where $h$ stands for the half-height of the channel. This was long enough to ensure convergence of phase-averaged statistics up to fourth-order moments.

The classical triple decomposition is used. A quantity $q$ is decomposed into a mean $\bar{q}$ an oscillating $\tilde{q}$ and fluctuating $q^{\prime}$ components. The angle brackets designate the phase averages, that is, $\langle q\rangle=\bar{q}+\tilde{q}$. The modulation characteristics of $\langle q\rangle$ are described by the amplitude $A_{\tilde{q}}$ and phase $\Phi_{\tilde{q}}$ of the fundamental mode. The relative amplitude $a_{\tilde{q}}=\left(A_{\tilde{q}} / \bar{q}\right)$ is also introduced for convenience.

\section{Rapid Distortion Model Strategy}

A review on rapid-distortion theory (RDT) can be found in Hunt and Carruthers. ${ }^{16}$ We will only describe here shortly some aspects of the RDT with a specific emphasis on its applications on shear flows.

The main hypothesis made in RDT is that the nonlinearity and moreover the viscosity can be neglected over timescales that are short relative to the decay timescales of the large-scale eddies. Consequently the fluctuating shear stresses are negligible compared to the distortion caused by the shear. The distortion time $T_{d}$ has to be short compared to the characteristic timescale $T_{L}=L /\left(u_{i}^{\prime} u_{i}^{\prime}\right)^{1 / 2}$ of the large eddies of scale $L$ to avoid their transformation and the energy transfer to the small scales. Thus, if $T_{d} \ll L /\left(u_{i}^{\prime} u_{i}^{\prime}\right)^{1 / 2}$ largescale structures have not enough time to go through the cascade transfer and generate nonlinearity by the vortex stretching, tilting, or rotation mechanisms. If in addition $T_{d}$ is small compared to the viscous timescale $T_{v}=L^{2} / \nu$, the viscosity can also be neglected during the distortion process. With these assumptions in mind, one can easily show that the fluctuating field of an homogeneous turbulent shear flow subject to a time-dependent velocity $\boldsymbol{u}(t, y)=[\beta(t) y, 0,0]$ is governed by

$$
\frac{\partial u_{j}^{\prime}}{\partial t}+\beta y \frac{\partial u_{j}^{\prime}}{\partial x_{1}}+u_{2}^{\prime} \beta \delta_{j, 1}=-\frac{1}{\rho} \frac{\partial p^{\prime}}{\partial x_{j}}
$$

where $u_{j}^{\prime}$ and $p^{\prime}$ are, respectively, the velocity and pressure fluctuations and $\delta_{i, j}$ is the Kronecker delta function. The last equation can be solved in Fourier space with appropriate initial conditions that depend essentially on the initial anisotropy. ${ }^{12}$

The rapid distortion theory is suitable for flows in time-space nonequilibrium. It deals with the history of the turbulence structure rather than the local dependence. Maxey ${ }^{13}$ considers the core axisymmetric structure of an internal turbulent flow with an initial isotropy parameter $s$ and which is subjected to a time-dependent shear of the form $u=[\beta(t) y, 0,0]$. He subsequently computes the velocity moments as a function of the distortion strain parameter

$$
\alpha(t)=\int_{0}^{t} \beta\left(t^{\prime}\right) \mathrm{d} t^{\prime}
$$

using the classical assumptions of RDT, namely, neglecting the nonlinear terms and moreover the viscosity. For large $T_{d}$ the distortion is essentially a slow phenomena, and the strain parameter takes the form $\alpha=T_{d}(\partial u / \partial y)$, while for short times one has clearly $\partial \alpha / \partial t=\partial u / \partial y$. Maxey ${ }^{13}$ proposes to couple these equations into a first-order model $\partial \alpha / \partial t=-\alpha / T_{d}+\partial u / \partial y$ that correctly predicts the short $\left(t \ll T_{d}\right)$ and long $\left(t \gg T_{d}\right)$ time behaviors. He observes furthermore that "the developed turbulent structure in channel or pipe flows is similar to that of a truncated rapid distortion stopped when $\alpha$ has settled to a local equilibrium effective value." The matching of the distributions of the structural parameters such as $-\overline{u^{\prime} v^{\prime}} / \bar{k}$, or $\overline{u^{\prime} u^{\prime}} / \bar{k}$ computed as a function of $\alpha(t)$ with the turbulent internal flow data, allows the determination of an effective strain parameter $\alpha_{\text {eff }}(y)$ and a local distortion timescale $T_{d}(y)=\alpha_{\text {eff }}(y) /(\partial \bar{u} / \partial y)$. The latter reveals the existence of a relaxation timescale of large eddies that enters into first-order models in unsteady forced turbulent flows. This will be detailed in the next section. Note that the validity of the method lies on the similarity of the structural parameters with a truncated rapid distortion model in internal shear flows. This is just an observation, and there is no a priori justification of this procedure for more general types of distortions.

Figure 2 shows the distribution of $-\overline{u^{\prime} v^{\prime}} / \bar{k}=F\left(\alpha_{\text {eff }}\right)$ for two values of the initial isotropy parameter $s=2 \overline{u^{\prime} u^{\prime}} /\left(\overline{v^{\prime} v^{\prime}}+\overline{w^{\prime} w^{\prime}}\right)$ defined as the ratio of the moments at the centerline. This distribution, combined with the profiles $-\overline{u^{\prime} v^{\prime}}$ and $\bar{k}$ obtained through standard steady $k-\omega$ closure, gives $\alpha_{\text {eff }}(y)$ and $T_{d}(y)$. The standard $k-\omega$ scheme results in $s=1.2$, a value that differs only slightly from 1.1 of Laufer's ${ }^{17}$ experimental data. The variation of $s$ from 1.2 to 1.5 did not change appreciably the modulation characteristics. We did not investigate the effect of larger, unrealistic isotropy parameters. The near-wall distribution of the effective strain parameter has not been clearly addressed in past studies: $\alpha_{\mathrm{eff}}(y)$ is determined from the bench experimental data from the centerline to the low log layer and extrapolated to the wall. The structural parameters $-\overline{u^{\prime} v^{\prime}} / \vec{k}$ or $\overline{u^{\prime} u^{\prime}} / \bar{k}$ vary rapidly in the buffer and viscous sublayers, and the

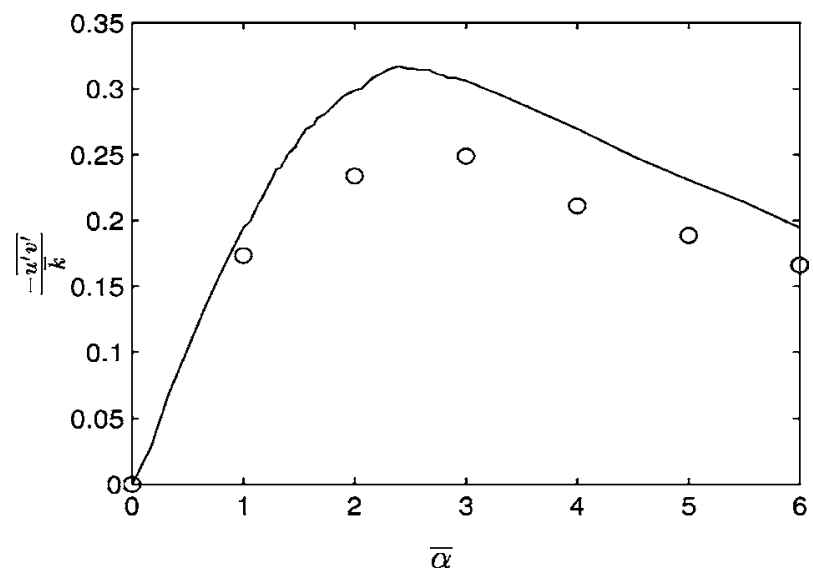

Fig. 2 Ratio of the shear stress to the kinetic energy vs the effective strain parameter according to $\mathrm{Maxey}^{13}:$ — , initial isotropy parameter $s=1.2$ and $\circ, s=1.5$. 


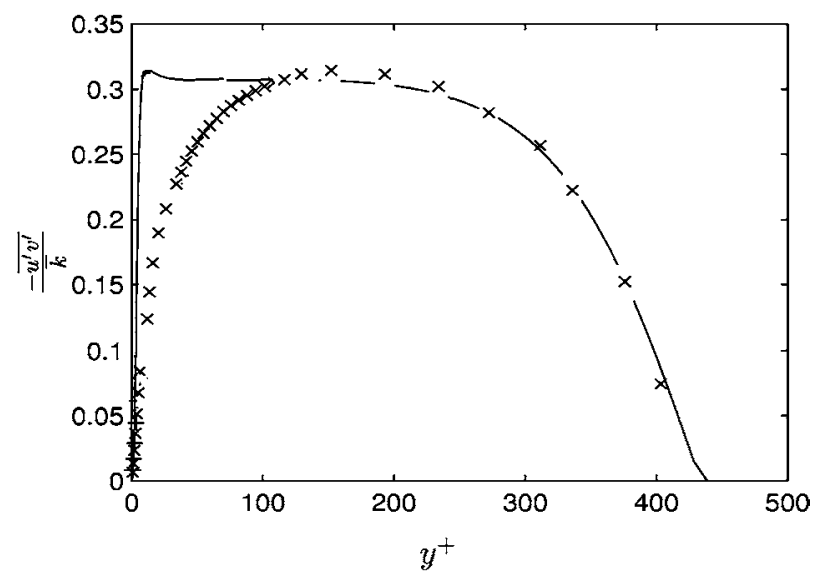

Fig. 3 Distribution of the ratio of the shear stress to the kinetic energy vs the wall-normal distance obtained by the steady $k-\omega$ model: - , $k-\omega$ without low Reynolds effects and $\times$, obtained through $k-\omega$ with the $\boldsymbol{R e}_{t}$ correction.

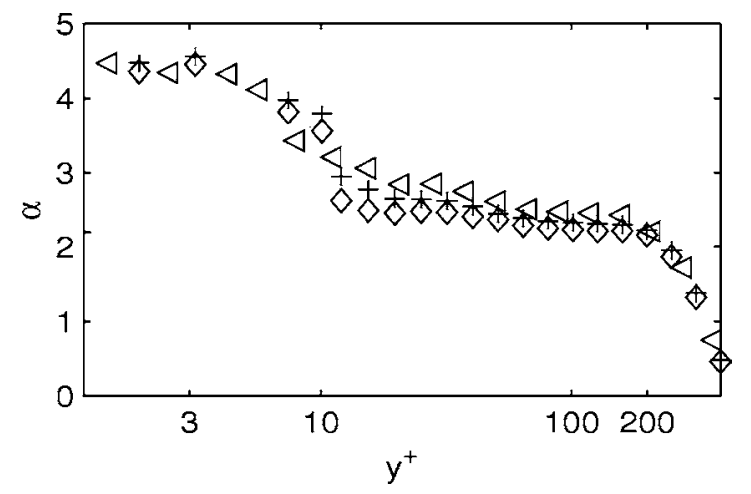

Fig. 4 Time-mean effective strain parameter vs the distance to the wall in wall units: + , steady flow; $\triangleleft$, imposed frequency in wall units $f^{+}=$ 0.005 ; relative amplitude at the centerline $a_{\mathrm{u} \tilde{c}}=0.20$; and $\diamond, f^{+}=0.0003$, $a_{\mathrm{uc}}=\mathbf{0 . 2 0}$.

extrapolation is arbitrary. More importantly the truncated rapid distortion concept does not take into account the viscous effects. Thus, a consistent distribution of the effective distortion parameter has to be based on models that exclude near-wall viscous damping. This is achieved here by computing $\alpha_{\text {eff }}(y)$ from the $-\overline{u^{\prime} v^{\prime}} / \bar{k}$ profile obtained by $k-\omega$ without low-Reynolds-number effects (Fig. 3). The modeling of viscous damping is performed separately through the turbulent Reynolds-number correction (see the next section). Figure 4 shows the resulting distribution of the effective strain parameter under steady and some unsteady flow conditions. It is seen that in the later case the long time-average $\bar{\alpha}_{\text {eff }}$ distribution coincides with that of the stationary flow, indicating that the time-mean quantities are unaffected by the imposed unsteadiness. That will be later discussed in further detail. The effective strain parameter is zero at the centerline and nearly constant in $10 \leq y^{+} \leq 200$. At $y^{+} \leq 10$, $-\overline{u^{\prime} v^{\prime}} / \bar{k}$ decreases rapidly to zero, and $\alpha_{\text {eff }}(y)$ should increase towards unrealistically large values, according to the right part of the curve given in Fig. 3. However, the concept of the RDT as to obtain an asymptotic structure for the turbulence in a mean shear suggests a finite distortion timescale and a finite distortion strain close to the wall. Several $\alpha_{\text {eff }}(y)$ distributions in the low buffer and viscous layers have been tried. ${ }^{18}$ Best agreement with the experiments has been obtained by assuming a finite effective distortion parameter of 4.5 at $y^{+} \leq 7$ resulting in a relaxation time $T_{d}^{+}=4.5$ in the viscous sublayer. The resulting distortion timescale distribution is not significantly different from that used by MK (Fig. 5). This is noteworthy because the methodology in the present study is different. MK use the experimental profiles of the structural parameter $-\overline{u^{\prime} v^{\prime}} / \overline{u^{\prime} u^{\prime}}$, whereas the output of the standard $k-\omega$ scheme and $-\overline{u^{\prime} v^{\prime}} / \bar{k}$ distribution are used here.

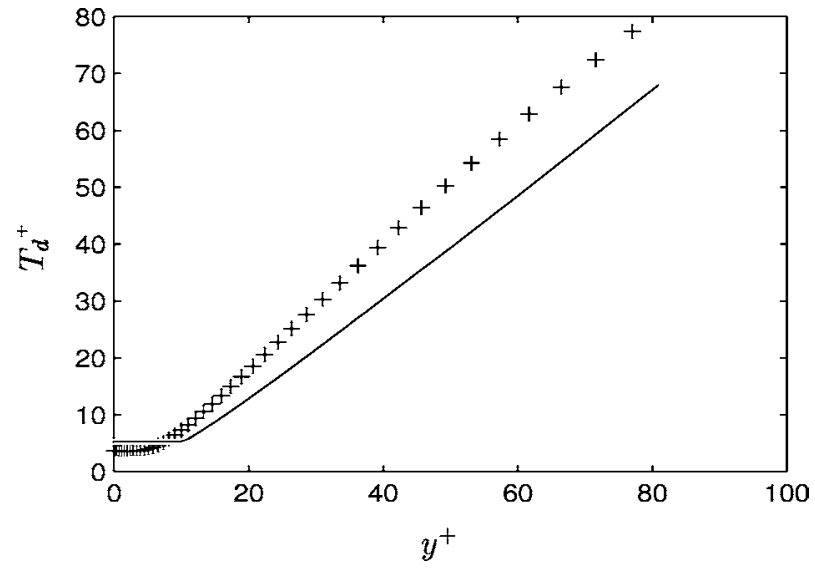

Fig. 5 Distortion timescale vs the wall-normal distance: - - , distribution used in this study and + , correspond to the profile used in Mankbadi and Liu. ${ }^{9}$

\section{New Closure}

The direct application of the $k-\omega$ closure to the unsteady turbulent channel flow is

$$
\begin{gathered}
\frac{\partial\langle\omega\rangle}{\partial t}=-\langle\gamma\rangle \frac{\langle\omega\rangle}{\langle k\rangle}\left\langle u^{\prime} v^{\prime}\right\rangle \frac{\partial\langle u\rangle}{\partial y}-\beta\langle\omega\rangle^{2}+\frac{\partial}{\partial y}\left[\left(v+\sigma\left\langle v_{t}\right\rangle\right) \frac{\partial\langle\omega\rangle}{\partial y}\right] \\
\frac{\partial\langle k\rangle}{\partial t}=-\left\langle u^{\prime} v^{\prime}\right\rangle \frac{\partial\langle u\rangle}{\partial y}-\left\langle\beta^{\prime}\right\rangle\langle\omega\rangle\langle k\rangle+\frac{\partial}{\partial y}\left[\left(v+\sigma\left\langle v_{t}\right\rangle\right) \frac{\partial\langle k\rangle}{\partial y}\right]
\end{gathered}
$$

where $-\left\langle u^{\prime} v^{\prime}\right\rangle$ is the phase average of the Reynolds shear stress; $\langle\gamma\rangle, \sigma, \beta$, and $\left\langle\beta^{\prime}\right\rangle$ are numerical constants depending upon the turbulent Reynolds number $\left\langle R e_{t}\right\rangle=\langle k\rangle /\langle\omega\rangle ;\langle\rangle$ denotes the phase average; and $v$ is the cinematic viscosity. The rest of the symbols are usual, with $y$ standing for the wall normal distance, $u$ the streamwise velocity, $k$ the kinetic energy, $v_{t}$ the eddy viscosity, and $t$ the time. According to Wilcox, ${ }^{11}$ the coefficients in Eqs. (1) and (2) are related to the turbulent Reynolds number by

$$
\begin{aligned}
& \langle\gamma\rangle=\frac{5}{9} \frac{1 / 10+\left\langle R e_{t}\right\rangle / R_{\omega}}{1+\left\langle R e_{t}\right\rangle / R_{\omega}}(\langle\chi\rangle)^{-1} \\
& \left\langle\beta^{\prime}\right\rangle=\frac{9}{100} \frac{5 / 18+\left(\left\langle R e_{t}\right\rangle / R_{\beta}\right)^{4}}{1+\left(\left\langle R e_{t}\right\rangle / R_{\beta}\right)^{4}}
\end{aligned}
$$

with

$$
\langle\chi\rangle=\frac{1 / 40+\left\langle R e_{t}\right\rangle / R_{k}}{1+\left\langle R e_{t}\right\rangle / R_{k}}
$$

The ensemble of the numerical constants appearing in Eqs. (1-3) is as follows: $\sigma=0.5, \beta=3 / 40, R_{\omega}=2.7, R_{\beta}=8$, and $R_{k}=6$ (Ref. 11). The adjustment of these coefficients is based on the modeling of the transition in a turbulent boundary layer. The effect of $\left\langle R e_{t}\right\rangle$ is mainly confined in the buffer and low log layer.

Equations (1) and (2) are combined with the Boussinesq relationship:

$$
-\left\langle u^{\prime} v^{\prime}\right\rangle=\left\langle v_{t}\right\rangle \frac{\partial\langle u\rangle}{\partial y}
$$

and the entire closure is achieved by relating the eddy viscosity to $\langle k\rangle$ and $\langle\omega\rangle$ by

$$
\left\langle v_{t}\right\rangle=\langle\chi\rangle \frac{\langle k\rangle}{\langle\omega\rangle}
$$

Clearly, this approach is quasi-steady, and therefore its validity is restricted to low-frequency imposed oscillations. Equations (1) and 
(2) are "exact," but Eq. (4) supposes time equilibrium. The rapid distortion model is therefore introduced in a way similar but not identical to MK. To this end, the effective strain parameter $\left\langle\alpha_{\text {eff }}\right\rangle$ governed by

$$
\frac{\partial\left\langle\alpha_{\mathrm{eff}}\right\rangle}{\partial t}=-\frac{\left\langle\alpha_{\mathrm{eff}}\right\rangle}{T_{d}(y)}+\frac{\partial\langle u\rangle}{\partial y}+\frac{\partial}{\partial y}\left[D(y) \frac{\partial\left\langle\alpha_{\mathrm{eff}}\right\rangle}{\partial y}\right]
$$

is used, according to Maxey ${ }^{13}$ and where $T_{d}$ stands for the distortion timescale. Note that the diffusion of the effective strain is also taken into account through the last term as suggested by Townsend. ${ }^{12}$ The diffusion coefficient $\langle D(y)\rangle$ is taken equal to the eddy viscosity $\left\langle v_{t}(y)\right\rangle$ according to the Townsend's proposition. Subsequently, the cyclic modulations of the Reynolds shear stress and of the eddy diffusivity are computed according to

$$
\begin{gathered}
\frac{-\left\langle u^{\prime} v^{\prime}\right\rangle}{\langle k\rangle}=F\left(\left\langle\alpha_{\mathrm{eff}}\right\rangle\right) \\
\left\langle v_{t}\right\rangle=\langle\chi\rangle F\left(\left\langle\alpha_{\mathrm{eff}}\right\rangle\right) \frac{\langle k\rangle}{\partial\langle u\rangle / \partial y}
\end{gathered}
$$

where the function $F\left(\left\langle\alpha_{\text {eff }}\right\rangle\right)$ together with the distortion timescale $T_{d}(y)$ are obtained by the bench data of the steady turbulent channel as we discussed in the preceding section.

Central finite difference schemes of order two are used for spatial terms. A hybrid scheme is used for time discretization. It is implicit for the diffusion terms and is explicit and implicit for the nonlinear terms when they are respectively positive and negative. This scheme is unconditionally stable according to Wilcox. ${ }^{11}$ Two-hundred-fifty modes are used in the wall-normal direction in a nonuniform grid and the closest computational point to the wall is at 0.1 wall unit. The mesh number was varied between 100 and 500 without significant modifications in the results. The time step varied between 0.5 to 0.7 wall units. The terms in $\alpha$ of the effective strain parameter equation are discretized by a Crank-Nicholson scheme, whereas for the shear term of this equation we used an Adams-Bashforth discretization. We tried a sixth-order Adams-Bashforth scheme for $\alpha$ discretization but obtained sensibly the same results with a Crank-Nicholson scheme that consequently was adopted.

In a previous work, MK uses a simplified version of the turbulent kinetic energy transport equation through

$$
\frac{\partial \tilde{k}}{\partial t}=-u^{\prime} \tilde{v}^{\prime} \frac{\partial \bar{u}}{\partial y}-\overline{u^{\prime} v^{\prime}} \frac{\partial \tilde{u}}{\partial y}+v \frac{\partial^{2} \tilde{k}}{\partial y^{2}}-\tilde{\varepsilon}
$$

where $\tilde{\varepsilon}$ is the modulation of the viscous dissipation rate. Clearly the pressure-velocity strain and the triple correlations are neglected arguing that the nonlinear effects are negligible near the wall. Furthermore, the mean quantities such as $\bar{u},-\overline{u^{\prime} v^{\prime}}$, etc., are supposed to not be affected by the imposed unsteadiness, and the standard distributions are used as input in the closure scheme. The most restrictive hypothesis they use concerns the dissipation. They first suppose local time equilibrium and more restrictively quasi-steadiness between the dissipation and the production. Then, they hypothesize the same eddy viscosity for the mean and modulated stresses leading finally to

$$
\tilde{\varepsilon}=-2 u^{\prime} \tilde{v}^{\prime} \frac{\partial \bar{u}}{\partial y}
$$

The closure is achieved by linearization of the equations coupled with the simplified form of Eqs. (5) and (6). This approach deals with essentially small perturbations, and the authors argue that a linear description suffices in this case. Yet, it is well established that the modulation characteristics properly scaled with the imposed amplitude do not depend on the latter and that the local time equilibrium is more than questionable in the high-frequency regime.

As already pointed out, this unsteady closure scheme is similar to that of MK. There are, however, important differences:

1) There is no hypothesis of any kind on the effect of the imposed unsteadiness on the time mean flow characteristics here, while MK suppose that the time mean flow is unaffected.
2) MK use a linear version of the rapid distortion equation (5), while the relationships (1), (2), and (6) are exact and can be used for large imposed amplitudes here.

3) MK suppose that there is time equilibrium between the turbulence energy and dissipation near the wall, while any hypothesis of this kind is made here.

4) Finally we also introduce the gradient-type diffusion term in the effective strain equation (2) and take into account the transport of $\left\langle\alpha_{\text {eff }}\right\rangle$ by small-scale turbulence.

We numerically have shown that the $k-\omega$ equations (1) and (2) are compatible with the rapid distortion equations (5) and (6) in unsteady turbulent channel flow and unsteady boundary layers with or without adverse pressure gradients as well. ${ }^{18}$

\section{Results and Discussion}

One of the aims of the present study is to compare experimental and modeled wall shear-stress intensity modulation characteristics $\left\langle\tau^{\prime} \tau^{\prime}\right\rangle$. The wall shear-stress intensity is always under the direct effect of the oscillating shear whatever is the imposed frequency. Thus, the responses of the amplitude and phase of $\left\langle\tau^{\prime} \tau^{\prime}\right\rangle$ are crucial in unsteady wall flows. The direct determination of the wall shear-stress intensity is impossible with $k-\omega / \mathrm{RD}$ method. Yet, one can obtain a good estimation by analyzing the asymptotic behavior of $\langle k\rangle /\langle u\rangle^{2}$ close to the wall. The streamwise, wall-normal, and spanwise velocity components behave, respectively, as

$u^{\prime} \sim y+\mathcal{O}\left(y^{2}\right), \quad v^{\prime} \sim y^{2}+\mathcal{O}\left(y^{3}\right), \quad w^{\prime} \sim y+\mathcal{O}\left(y^{2}\right)$

and consequently $\langle k\rangle=\frac{1}{2}\left\{\left\langle u^{\prime} u^{\prime}\right\rangle+\left\langle w^{\prime} w^{\prime}\right\rangle\right\}+\mathcal{O}\left(y^{4}\right)$ as $y \rightarrow 0$. Furthermore, next to the wall,

$$
\left\langle u^{\prime} u^{\prime}\right\rangle \approx\left\langle\omega_{z 0}^{\prime} \omega_{z 0}^{\prime}\right\rangle y^{2}, \quad\left\langle w^{\prime} w^{\prime}\right\rangle \approx\left\langle\omega_{x 0}^{\prime} \omega_{x 0}^{\prime}\right\rangle y^{2}
$$

where $\omega_{z 0}^{\prime}$ and $\omega_{x 0}^{\prime}$ are, respectively, the fluctuating spanwise and streamwise vorticity components at the wall. Thus,

$$
\begin{aligned}
& \langle k\rangle=\frac{\left\langle u^{\prime} u^{\prime}\right\rangle}{2}\left(1+\frac{\left\langle\omega_{x 0}^{\prime} \omega_{x 0}^{\prime}\right\rangle}{\left\langle\omega_{z 0}^{\prime} \omega_{z 0}^{\prime}\right\rangle}\right)+\mathcal{O}\left(y^{4}\right) \\
& =\frac{\left\langle u^{\prime} u^{\prime}\right\rangle}{2}\left[1+\overline{\overline{\omega_{x 0}^{\prime} \omega_{x 0}^{\prime}}}\left(\frac{1+\Omega_{\omega^{\prime} x 0}}{\overline{\omega_{z 0}^{\prime} \omega_{z 0}^{\prime}}}\right)\right]+\mathcal{O}\left(y^{4}\right)
\end{aligned}
$$

where $\tilde{\Omega}$ stands for relative modulation and reads for

$$
\Omega_{\tilde{q}}=\tilde{q q} / q q
$$

In the quasi-steady limit characterized by significantly slow modulations, one has exactly

$$
\langle k\rangle_{\mathrm{qs}}=\frac{\left\langle u^{\prime} u^{\prime}\right\rangle}{2}\left(1+\frac{\overline{\omega_{x 0}^{\prime} \omega_{x 0}^{\prime}}}{\overline{\omega_{z 0}^{\prime} \omega_{z 0}^{\prime}}}\right)
$$

In the medium and high imposed frequency range, and the limit of small imposed amplitudes,

$$
\langle k\rangle \approx \frac{\left\langle u^{\prime} u^{\prime}\right\rangle}{2}\left\{1+\frac{\overline{\omega_{x 0}^{\prime} \omega_{x 0}^{\prime}}}{\overline{\omega_{z 0}^{\prime} \omega_{z 0}^{\prime}}}\left[1+\tilde{\Omega}_{\omega^{\prime} x 0}-\tilde{\Omega}_{\omega^{\prime} z 0}\right]\right\}+\mathcal{O}\left(y^{4}\right)
$$

The stress ratios such as $-\left\langle u^{\prime} v^{\prime}\right\rangle /\left\langle u^{\prime} u^{\prime}\right\rangle$ or $\left\langle v^{\prime} v^{\prime}\right\rangle /\left\langle u^{\prime} u^{\prime}\right\rangle$ are only slightly modulated in near-wall unsteady flows. ${ }^{19}$ Although measurements of $\left\langle w^{\prime} w^{\prime}\right\rangle /\left\langle u^{\prime} u^{\prime}\right\rangle$ are nowhere reported in the literature, it is likely that this specific structural parameter is also slightly modulated implying that $\tilde{\Omega}_{\omega^{\prime} x 0} \approx \tilde{\Omega}_{\omega^{\prime} z 0}$. Because $\left\langle u^{\prime} u^{\prime}\right\rangle /\langle u\rangle^{2}$ tends asymptotically to $\left\langle\tau^{\prime} \tau^{\prime}\right\rangle /\langle\tau\rangle^{2}$ as $y \rightarrow 0$, one has

$$
\frac{\left\langle\tau^{\prime} \tau^{\prime}\right\rangle}{\langle\tau\rangle^{2}} \approx \frac{2}{1+\overline{\omega_{x 0}^{\prime} \omega_{x 0}^{\prime}} / \overline{\omega_{z 0}^{\prime} \omega_{z 0}^{\prime}}} \frac{\langle k\rangle}{\langle u\rangle^{2}}
$$

Because the time-mean flow is unaffected by imposed unsteadiness as revealed by the ensemble of experimental studies and $\overline{\omega_{x 0}^{\prime} \omega_{x 0}^{\prime}} / \overline{\omega_{z 0}^{\prime} \omega_{z 0}^{\prime}}=0.25$ according to Kim et al., ${ }^{20}$ one has

$$
\left\langle\tau^{\prime} \tau^{\prime}\right\rangle /\langle\tau\rangle^{2} \approx 1.6\langle k\rangle /\langle u\rangle^{2}
$$


The modulation characteristics of the wall shear stress are estimated from this last relationship and compared with the experiments.

There is no effect of the imposed unsteadiness on the time-mean flow characteristics, even when the imposed amplitude at the centerline is as large as $30 \%$ and the imposed frequency is near the bursting frequency of the base flow. This is in perfect agreement with the experiments. Figure 6 shows the time-mean profiles of the streamwise velocity, kinetic energy, shear stress, and dissipation in wall units obtained through $k-\omega$ and $k-\omega / \mathrm{RD}$ for two frequency parameters $l_{s}^{+}=8$ and 33. It is seen that both schemes result in unaffected timemean properties and that the profiles at the small-imposed amplitude $a_{\mathrm{uc}}=0.1$ collapse well with those obtained at $a_{\mathrm{uc}}=0.3$.

The $k-\omega$ scheme alone also predicts the modulation characteristics of the streamwise velocity $\langle u\rangle$ and the wall shear stress $\langle\tau\rangle$ well. Figure $7 \mathrm{a}$ shows the ratio of the wall shear-stress modulation amplitude $A_{\tilde{\tau}}$ to the amplitude $A_{\tilde{\tau} \text { Stokes }}=\sqrt{ } 2 \mu\left(A_{\mathrm{uc} \tilde{c}}\right) / l_{s}$ that a purely viscous Stokes flow would have. The results inferring from the modeling are compared both with the measurements and with MK. The imposed amplitude is $a_{\tilde{u} c}=0.20$ hereafter. It is seen that there is good agreement between the measurements and the predictions and that the MK model fails in the low-frequency (quasi-steady) regime. The numerical results inferred from the $k-\omega / \mathrm{RD}$ model are below one for $l_{s}^{+} \leq 15$, exactly as in the experiments. The phase shift $\Phi_{\tilde{\tau}}-\Phi_{\tilde{u} c}$ predicted by both $k-\omega$ and $k-\omega / \mathrm{RD}$ reaches a value of $45 \mathrm{deg}$ in the high-imposed frequency regime (Fig. 7b). There is clearly a coexistence of an unaffected time-mean flow with a purely oscillating viscous flow when the frequency is large enough. The amplitude and phase shift distributions of the measured and computed streamwise velocity oscillations $\langle u\rangle$ are also in close agreement (not shown here). The $k-\omega$ scheme alone predicts $A_{\tilde{u}}$ and $\Phi_{\tilde{u}}$ well and gives results quite close to the $k-\omega / \mathrm{RD}$ closure with only some subtle differences. ${ }^{18}$

The response of turbulence is particularly difficult to model in unsteady wall flows. The $k-\omega$ scheme alone fails considerably in predicting the amplitude and phase of fluctuating flow quantities. Figure 8a shows the ratio of the relative amplitude of the turbulent wall shear-stress intensity $\left\langle\tau^{\prime} \tau^{\prime}\right\rangle$ to the relative amplitude in the quasi-steady regime. The rapid decrease of the turbulence intensity modulation with the imposed frequency from the quasi-steady limit is clearly seen in this figure. This is because of the finite response time of the near-wall turbulence that cannot follow the imposed unsteadiness when the imposed timescale is smaller than its relaxation time. The decrease in $a_{\tau^{\prime} \tau^{\prime}}$ is accompanied with the apparition of large phase shifts $\Phi_{\tau^{\prime} \tau^{\prime}}-\Phi_{\tilde{U}_{c}}$, as shown in Fig. 8b. There is a good agreement between $k-\omega / \mathrm{RD}$ and the experiments, although the predictions are slightly larger at the highest imposed frequency investigated here. The agreement is also acceptable for the phase shifts. The time lag between $\left\langle\tau^{\prime} \tau^{\prime}\right\rangle$ and the centerline velocity oscillation is about 90 wall units. Such detailed comparisons are rather rare in the literature. Figure 8 shows that the relaxation is seriously underestimated by the $k-\omega$ model, which is not discussed here.

The high-frequency imposed oscillations are particularly interesting regarding the rapid distortion theory. Figure 9 shows the relative amplitude of the Reynolds shear-stress modulation at the imposed frequency $f^{+}=0.005$ where the modulations of the turbulent quantities decrease drastically, caused, once more, by the finite response time of the near-wall turbulence. This fact can clearly be understood by the direct comparisons of Fig. 9 with Fig. 10, which shows results in the quasi-steady regime. (Please be aware that the scales in the abscissas of these figures are different.) At $y^{+}=20$ for example, the shear stress is nearly eight times less modulated at $f^{+}=0.005$ than in the quasi-steady regime. The decrease of the $-\left\langle u^{\prime} v^{\prime}\right\rangle$ modulation is accompanied by large phase shifts, and the time lag $\Delta t^{+}=\left(\Phi_{-u^{\prime} \tilde{v}^{\prime}}-\Phi_{-u \tilde{c}}\right) l_{s}^{+2} / 2$ decreases in the inner layer. Tardu et al. (Ref. 4, p. 134) related this decrease to the diffusion of the modulation of fluctuating quantities away from the wall with a diffusivity equal to the diffusivity of time-mean turbulent kinetic energy. The $k-\omega / \mathrm{RD}$ model agrees quantitatively well with the experimental data, not only for the amplitudes, but also for the phase shifts (Fig. 11).
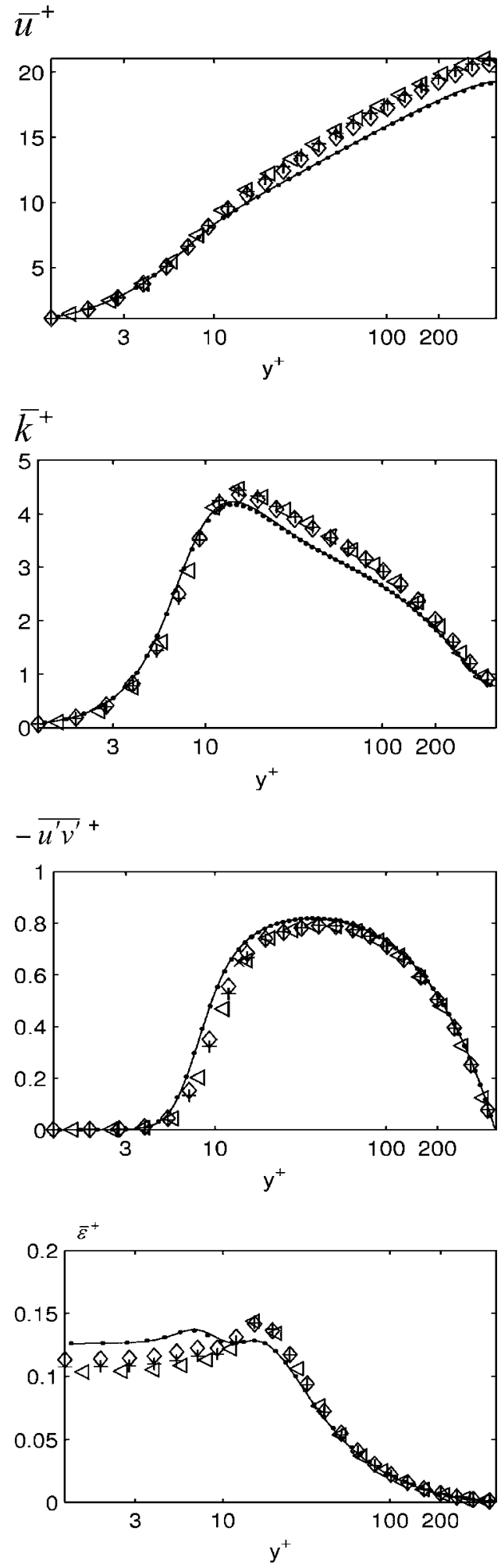

Fig. 6 Time-mean flow quantities. Mean velocity, kinetic energy, shear-stress and dissipation profiles: $k-\omega, a_{\mathrm{u} \tilde{\mathrm{c}}}=0.3 ; \ldots, l_{s}^{+}=33$; $l_{s}^{+}=8 k-\omega / \mathrm{RD}, a_{\mathrm{uc} \tilde{c}}=0.3 ; \diamond, l_{s}^{+}=33 ; \triangleleft, l_{s}^{+}=8, k-\omega / \mathrm{RD}, a_{\mathrm{uc} \tilde{c}}=0.1$; and + , $l_{s}^{+}=8$.

The agreement is almost perfect for a large range of imposed frequencies in the whole layer. We show in Fig. 12 the modulation characteristics of the Reynolds shear stress at $y^{+}=15$ and at roughly the beginning of the $\log$ layer $y^{+}=40$ to illustrate the success of the closure. The amplitude [Eq. (12a)] and phase shifts [Eq. (12b)] of, respectively, the Reynolds shear stress, the kinetic energy, the dissipation, and production are compared with the measurements when they are available. We only could measure $\left\langle u^{\prime} u^{\prime}\right\rangle$ and $\left\langle v^{\prime} v^{\prime}\right\rangle$. 


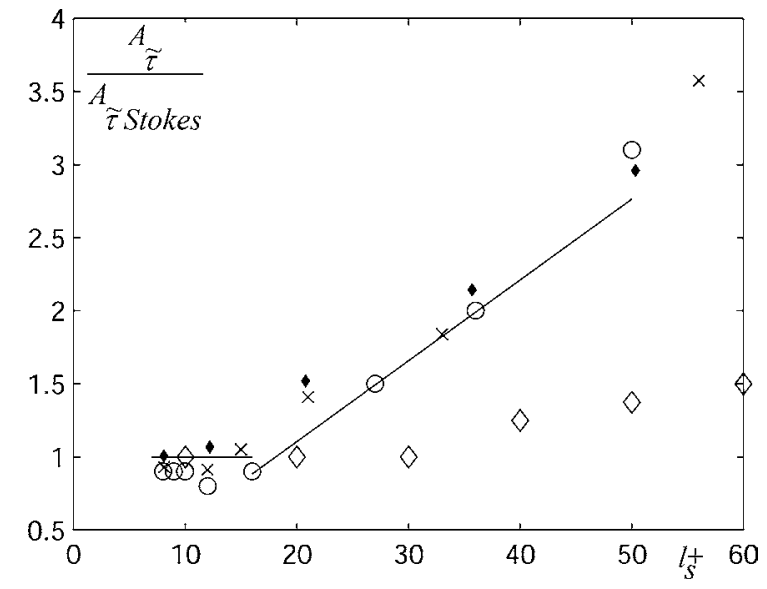

a)

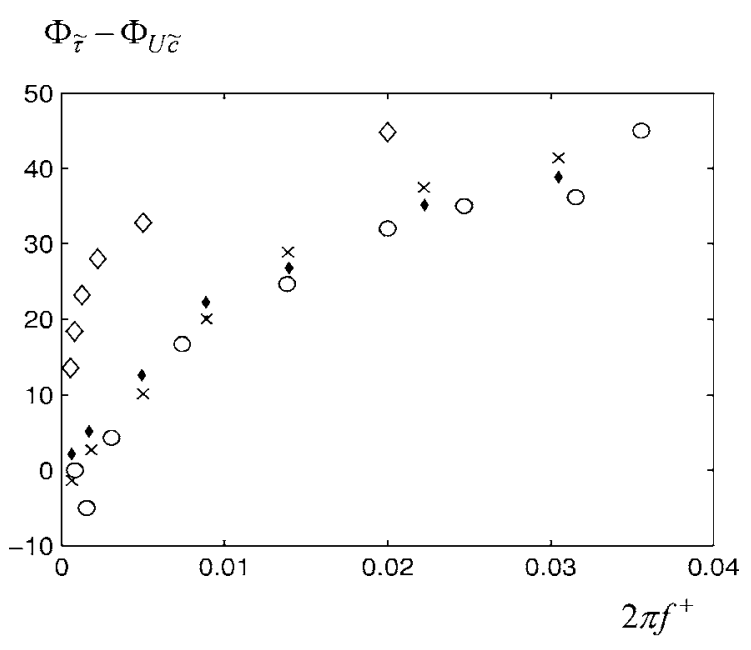

b)

Fig. 7 Wall shear-stress modulation vs the Stokes length parameter: a) amplitude (in wall units) and b) phase (frequency). The imposed amplitude is $30 \%$ of the centerline velocity: $\times, k-\omega / \mathrm{RD} ; \diamond, k-\omega ; \circ$, and $\diamond$, Mankbadi and Liu. ${ }^{9}$

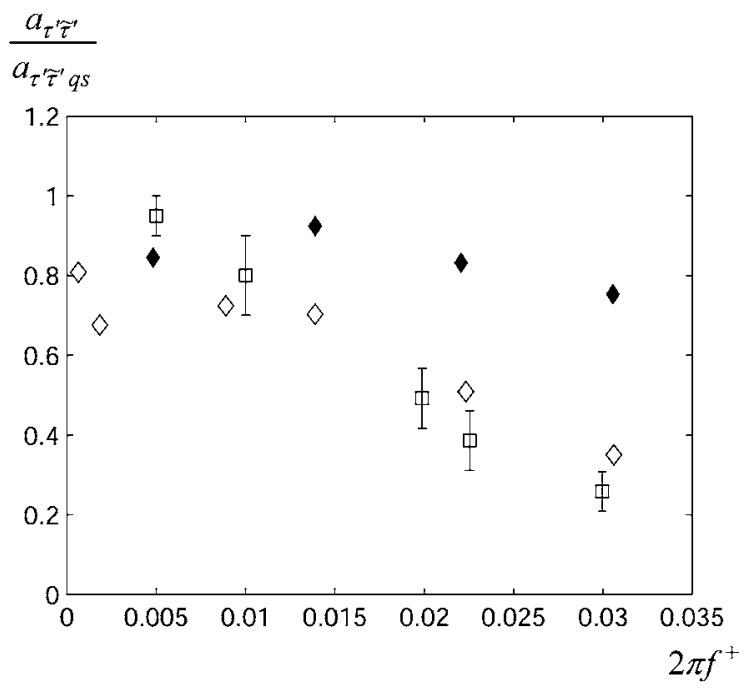

a)

Fig. 8 Modulation characteristics of the wall shear-stress turbulent intensity: $\diamond, k-\omega / R D ; \diamond, k-\omega$; and $\Phi$, measurements with dispersion symbols. a) Relative amplitude divided by the relative amplitude in the quasi-steady regime. It can easily be shown that $a_{\tau^{\prime} \tilde{\tau}^{\prime} \mathrm{qs}} \approx 4 a_{\mathrm{uc}}$ in the small imposed amplitude approximation. b) Phase shift with respect to the centerline velocity oscillations.

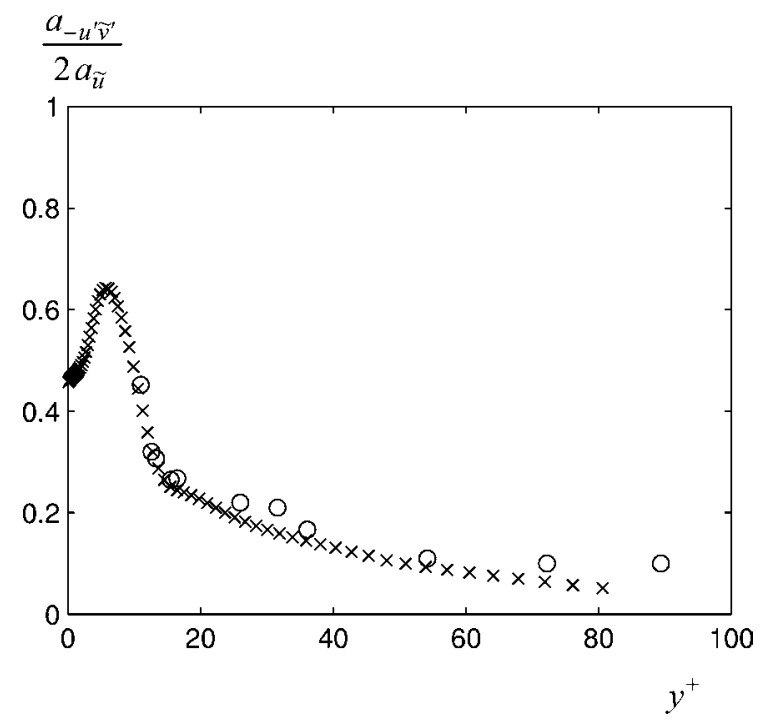

Fig. 9 Amplitude of the shear stress at $l_{s}^{+}=8: \circ$, measurements and $x$, predicted values.

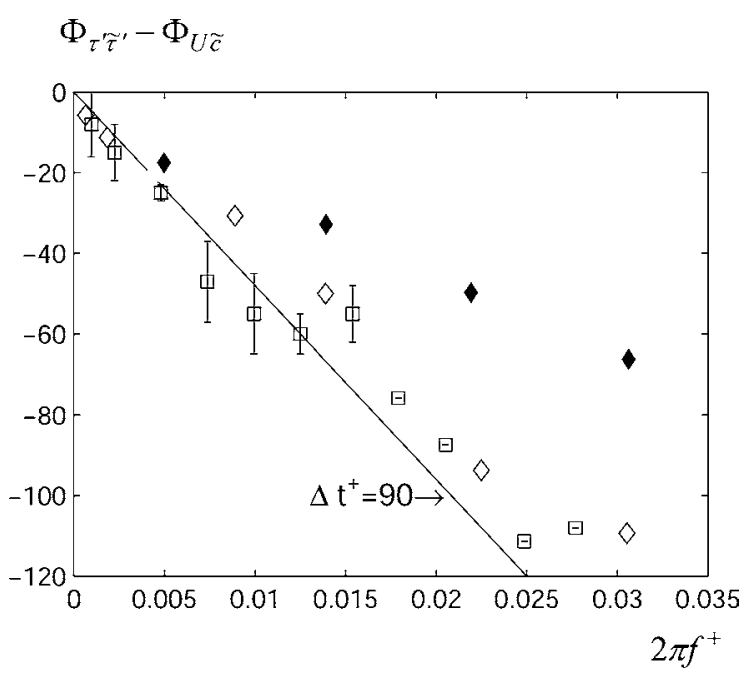

b) 


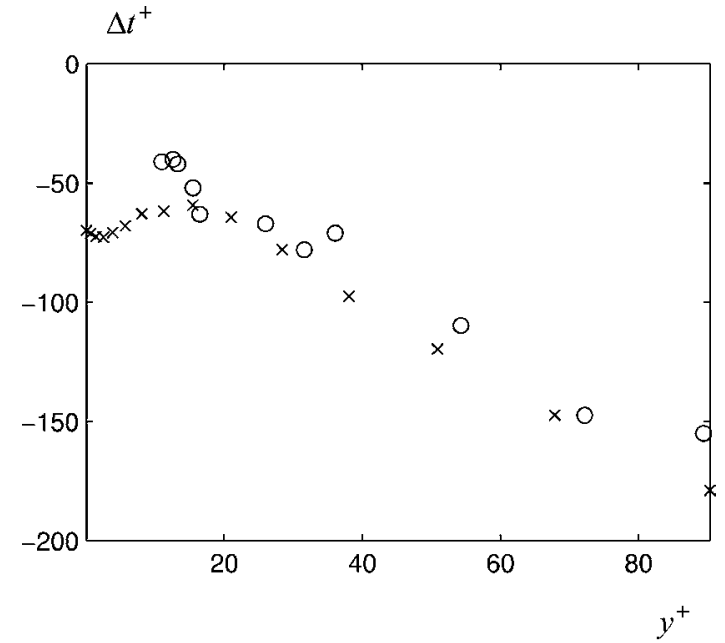

Fig. 11 Time lag of the shear-stress modulation in the inner layer at $l_{s}^{+}=8$. The symbols are identical to those in Fig. 9 .
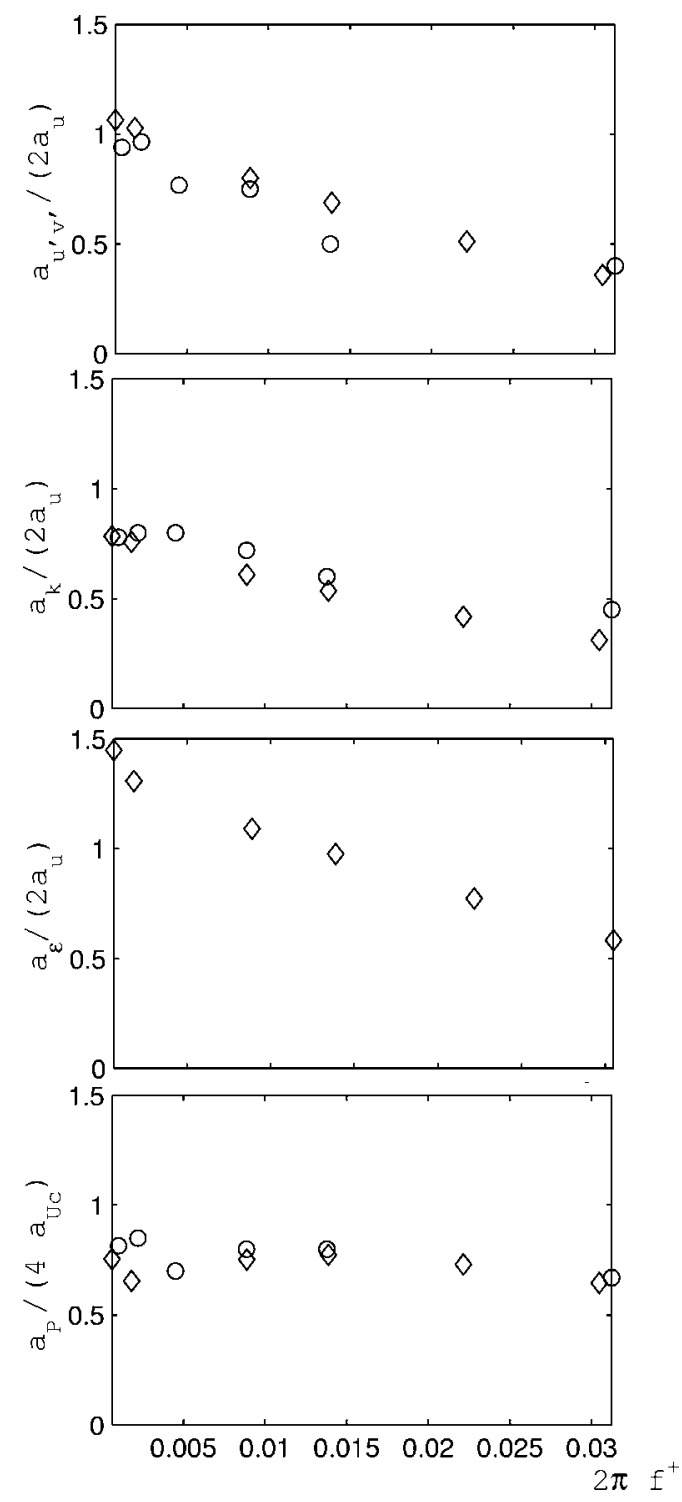

a)

Fig. 12 Modulation characteristics of turbulent flow quantities vs the imposed frequency: $\odot$, measurements and $\diamond, k-\omega /$ RD. Amplitudes: a and b) at, respectively, $y^{+}=15$ and $y^{+}=40 ; \mathrm{c}$ and d) at, respectively, $y^{+}=15$ and $y^{+}=40$.
The computed kinetic energy modulation $\langle k\rangle$ is thus compared with its major contribution $\frac{1}{2}\left\langle u^{\prime} u^{\prime}\right\rangle$. The success of $k-\omega / \mathrm{RD}$ model in the whole layer is surprisingly satisfactory.

Some remarks are necessary on the effect of the rapid distortion over the $k-\omega$ model alone with and without wall correction. We conducted several numerical experiments, by separately considering the simple quasi-steady $k-\omega$ models with and without $\left\langle R e_{t}\right\rangle$ correction. The following points have been noticed:

1) The quasi-steady closure compares well with the full model and the measurements in the log layer at $y^{+} \geq 50$ and in the whole relaxation regime $f^{+} \leq 0.005$.

2) In return, the rapid distortion plays an essential role in the low buffer and viscous sublayers, wherein the low Reynolds effects $\left\langle R e_{t}\right\rangle$ are also primordial. The $k-\omega$ closure alone overestimates the amplitude of the shear stress up to $60 \%$ in these zones and in the high-frequency regime.

3 ) The effect of the diffusion term in the transport equation of the effective strain parameter $\left\langle\alpha_{\text {eff }}\right\rangle$ is negligible.

To illustrate these observations, we show in Fig. 13 the distribution of the relative amplitude of the shear stress and kinetic energy modulations at $l_{s}^{+}=8$ obtained by the standard $k-\omega$ and $k-\omega / \mathrm{RD}$.
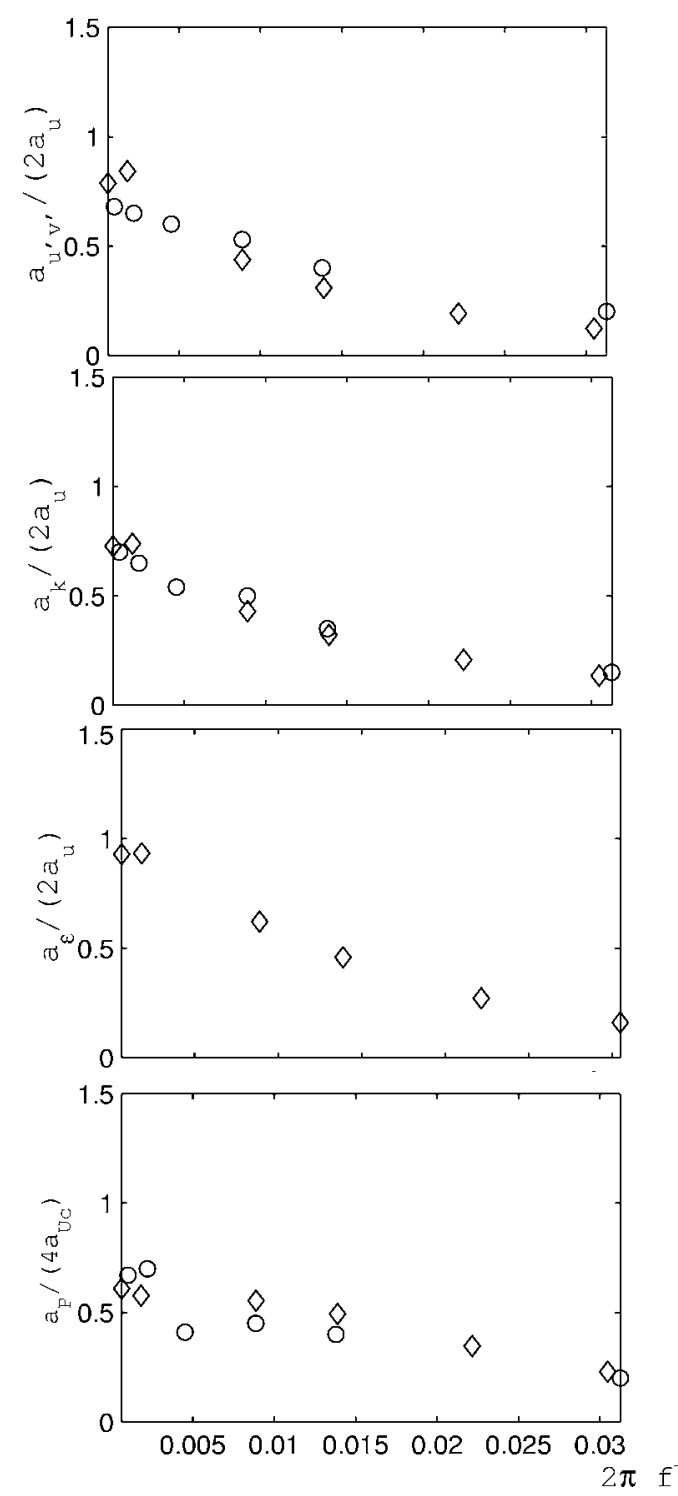

b) 

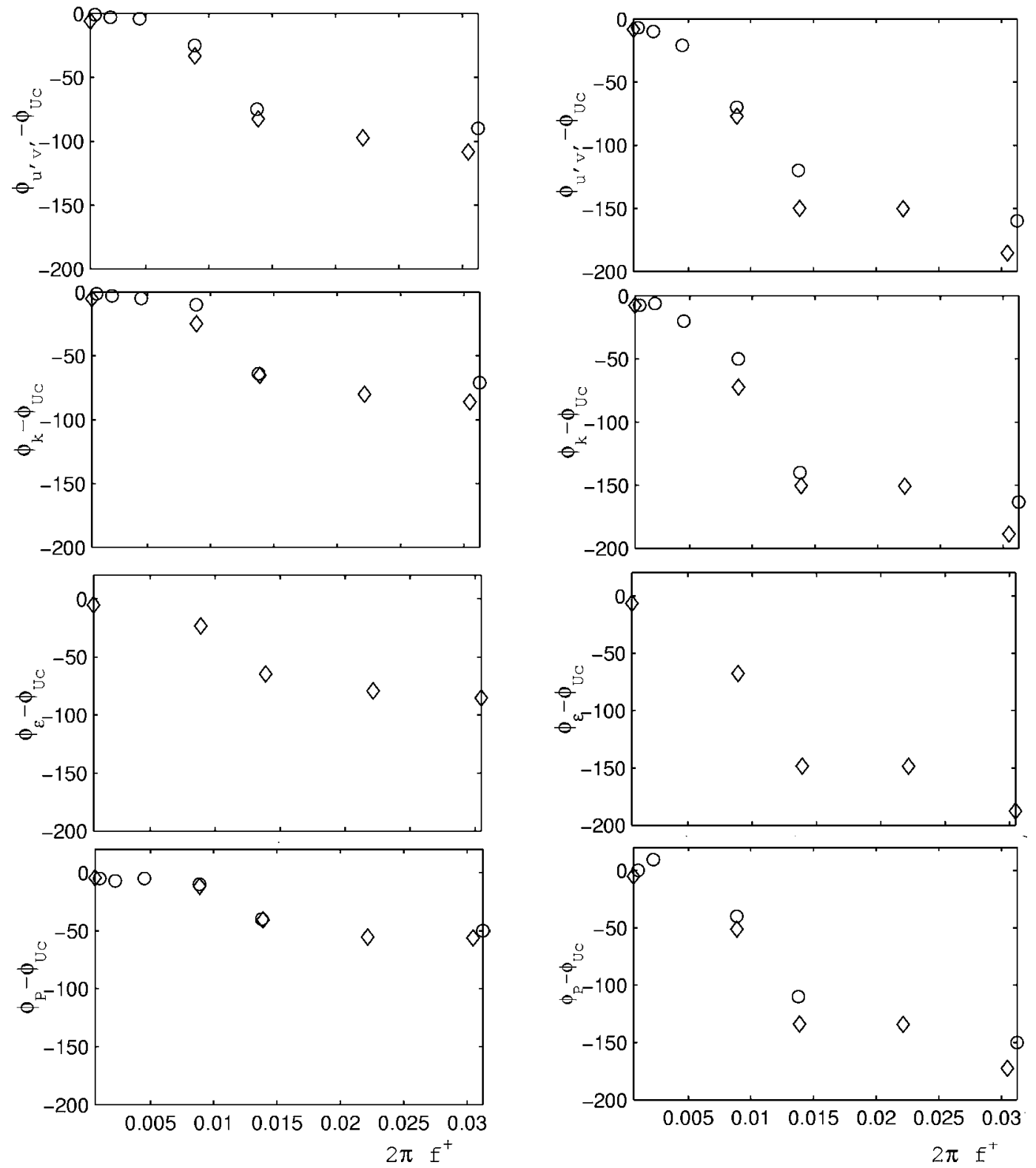

c)

d)

Fig. 12 Modulation characteristics of turbulent flow quantities vs the imposed frequency: $\bigcirc$, measurements and $\diamond, k-\omega /$ RD. Amplitudes: a and b) at, respectively, $y^{+}=15$ and $y^{+}=40 ; \mathrm{c}$ and d) at, respectively, $y^{+}=15$ and $y^{+}=40$ (continued).

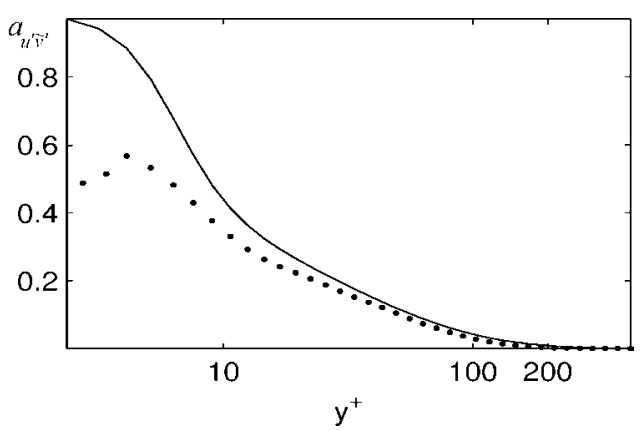

a)

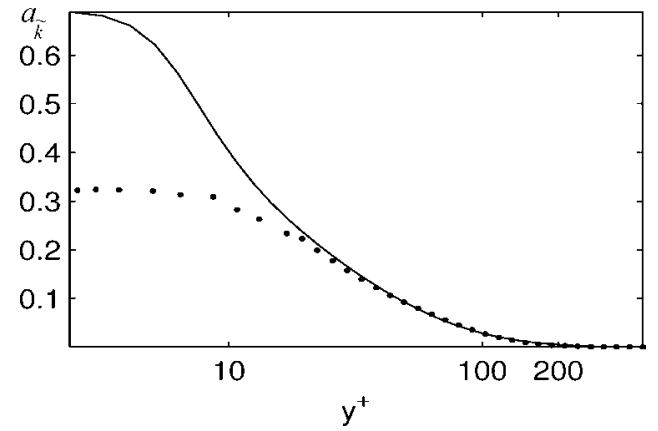

b)

Fig. 13 Distribution of the relative amplitudes of the a) shear stress and b) kinetic energy obtained by,$- k-\omega$ and $\cdots, k-\omega / \mathrm{RD}$ at $l_{s}^{+}=8$. The imposed amplitude is $a_{\mathrm{uc}}=0.20$. 
The modulation of the shear is constrained into the low buffer layer $y^{+}<2 l_{s}^{+}=16$ in this case, and the rest of the layer is in the plug flow regime. It is clearly seen in Fig. 13 that $k-\omega$ alone significantly overestimates the turbulent characteristic modulations in the oscillating shear zone because of its incapacity to correctly model the relaxation mechanism. In the plug flow layer $\left(y^{+} \gg 16\right)$, however, the closures give comparable results.

\section{Conclusions}

The one-point $k-\omega$ closure is combined with the rapid distortion theory to predict the modulation characteristics of fluctuating flow quantities in an unsteady channel flow subject to imposed velocity oscillations. The structural parameter $-\left\langle u^{\prime} v^{\prime}\right\rangle /\langle k\rangle$ is related to the effective strain parameter and injected into the classical $k-\omega$ relationships. The effective strain parameter modulation is the solution of a first-order linear differential equation containing the shear. The truncated rapid distortion concept does not take into account the near-wall viscous effects. To be consistent, the distortion timescale is determined by the results obtained through steady $k-\omega$ without low-Reynolds-number corrections. A finite effective strain parameter is attributed to the viscous sublayer. The classical interrelation between the eddy viscosity and $\langle k\rangle$ and $\langle\omega\rangle$ supposes time equilibrium and gives satisfactory results only in the low imposed frequency regime and/or in the logarithmic layer. This model fails in the zones wherein the shear modulations are significantly important, especially in the buffer and viscous sublayers. The $k-\omega / \mathrm{RD}$, in return, agrees quantitatively well with the experiments in the whole layer and the imposed frequency range. It is particularly efficient at the wall in predicting the amplitude and phase of the wall shear-stress turbulence intensity for $f^{+} \leq 0.005$.

There are few experimental data set for $f^{+} \geq 0.005$ that indicate a fully different reaction of the near-wall turbulence. The experimental data show that fluctuating shear stresses become modulated again, contrarily to the relaxation regime. ${ }^{15}$ There is no known model that gives satisfactory predictions, even qualitative at $f^{+} \geq 0.005$. We could obtain fairly good agreement between the predictions and the measurements by both forcing and phase shifting the turbulent Reynolds-number modulation. Although this procedure can be somewhat considered artificial, it has the merit to point at the primordial role played by the wall in the regeneration of the turbulence activity modulation. These results will be published elsewhere. It is finally asked here whether the future development of the model should not contain a supplementary term in the transport equation of the effective strain:

$$
\frac{\partial\left\langle\alpha_{\mathrm{eff}}\right\rangle}{\partial t}=-\frac{\left\langle\alpha_{\mathrm{eff}}\right\rangle}{T_{d}(y)}+\frac{\partial\langle u\rangle}{\partial y}+\frac{\partial}{\partial y}\left[D(y) \frac{\partial\left\langle\alpha_{\mathrm{eff}}\right\rangle}{\partial y}\right]+\frac{\partial}{\partial y}\left[\left\langle V \alpha_{\mathrm{eff}}\right\rangle\right]
$$

where both a gradient-type diffusion and convection by large-scale $\langle V\rangle$ motions are included. The modeling of the latter could, eventually, give better insight into the mechanism at very high imposed frequencies.

\section{References}

${ }^{1}$ Tu, S., and Ramaprian, B. R., "Fully Developed Periodic Turbulent Pipe Flow, Part 1, Main Experimental Results and Comparison with Predictions," Journal of Fluid Mechanics, Vol. 137, 1983, pp. 31-58.

${ }^{2}$ Mao, X., and Hanratty, T. J., "Studies of Wall Stress in a Turbulent Pulsating Pipe Flow," Journal of Fluid Mechanics, Vol. 194, 1986, pp. 15-44.

${ }^{3}$ Brereton, G. J., Reynolds, W. C., and Jarayaman, R., "Response of a Turbulent Boundary Layer to Sinusoidal Free Stream Unsteadiness," Journal of Fluid Mechanics, Vol. 221, 1990, pp. 131-163.

${ }^{4}$ Tardu, S., Binder, G., and Blackwelder, R., "Turbulent Channel Flow Subjected to Large Imposed Velocity Oscillations," Journal of Fluid Mechanics, Vol. 267, 1994, pp. 109-151.

${ }^{5}$ Fan, S., Laksminarayana, B., and Barnett, M., "A Low Reynolds Number $k-\varepsilon$ Model for Unsteady Turbulent Boundary Layer Flows," AIAA Journal, Vol. 31, No. 10, 1993, pp. 1777-1784.

${ }^{6}$ Chernobrovkin, A., and Lakshminarayana, B., "Development and Validation of Navier-Stokes Procedure for Turbomachinery Flow," AIAA Journal, Vol. 37, No. 5, 1999, pp. 557-563.

${ }^{7}$ Ekaterinaris, J. A., and Menter, F. R., "Computation of Oscillating Airfoil Flows with One and Two Equations Turbulence Models," AIAA Journal, Vol. 32, No. 12, 1994, pp. 2359-2053.

${ }^{8}$ Srinivasan, G. R., Ekaterinaris, J. A., and McCroskey, W. J., "Evaluation of Turbulence Models for Unsteady Flows of an Oscillating Airfoil," Computers and Fluids, Vol. 7, No. 7, 1995, pp. 833-861.

${ }^{9}$ Mankbadi, R. R., and Liu, J. T. C., "Near-Wall Response in Turbulent Shear Flows Subjected to Imposed Unsteadiness," Journal of Fluid Mechanics, Vol. 238, 1992, pp. 55-71.

${ }^{10}$ Greenblatt, D., "Hybrid Turbulence Model for Unsteady Boundary Layers," AIAA Journal, Vol. 36, No. 3, 1998, pp. 481-483.

${ }^{11}$ Wilcox, D. C., Turbulence Modeling for CFD, KNI, Inc., Anaheim, CA, 1998, pp. 73-170.

${ }^{12}$ Townsend, A. A., "Entrainment and the Structure of Turbulent Flow," Journal of Fluid Mechanics, Vol. 137, 1980, pp. 31-58.

${ }^{13}$ Maxey, M. R., "Distortion of Turbulence in Flows with Parallel Streamlines," Journal of Fluid Mechanics, Vol. 124, 1982, pp. 261-282.

${ }^{14}$ Lueptow, R. M., Breuer, K. S., and Haritonidis, J. H., "Computer-Aided Calibration of X Probes Using a Look Up Table," Experiments in Fluids, Vol. 6, 1988, pp. 115-118.

${ }^{15}$ Tardu, S., and Binder, G., "Response of Turbulence to Imposed Oscillations of High Frequency," Physics of Fluids, Vol. 5, No. 8, 1993, pp. 2028-2037.

${ }^{16}$ Hunt, J. C. R., and Carruthers, D. J., "Rapid Distortion Theory and the Problems of the Turbulence," Journal of Fluid Mechanics, Vol. 212, 1990, pp. 497-532.

${ }^{17}$ Laufer, J., "The Structure of Turbulence in Fully Developed Pipe Flow," NACA Rept. 1174, March 1954.

${ }^{18}$ Da Costa, P., "Application de la Distorsion Rapide à des Écoulements Turbulents Instationnaires," Ph.D. Dissertation, Dept. of Mechanics, Univ. Joseph Fourier, Grenoble, France, Oct. 2000.

${ }^{19}$ Feng, M., "Structure Fine de la Turbulence Dans les Écoulements Instationnaires en Canal," Ph.D. Dissertation, Dept. of Mechanics, Univ. of Grenoble, Grenoble, France, Sept. 1993.

${ }^{20}$ Kim, J., Moin, P., and Moser, R., "Turbulence Statistics in Fully Developed Channel Flow at Low Reynolds Number," Journal of Fluid Mechanics, Vol. 177, 1987, pp. 133-166. 